\title{
Default Model Network Mechanism in Current and Remitted Depression
}

\author{
Qin Dai ${ }^{1-3}$, Xuntao Yin ${ }^{4}$, Jian Huang ${ }^{5}$, Hong $\mathrm{Li}^{1, \dagger}$, Jian Wang ${ }^{4, \dagger}$, Zhengzhi Feng ${ }^{2,+}$
}

\begin{abstract}
Objective: An altered default mode network (DMN) in the patients with major depressive disorder (MDD) has been frequently reported, which has been improved after treatment. A recent study has reported abnormal function connectivity between the DMN and the central executive network (CEN) in depression, however, whether this disturbed connectivity involves in the remission of depression remains unclear.
\end{abstract}

Methods: 30 patients with MDD and 27 individuals with the remitted depression (RMD) were recruited. All of them received a brain functional magnetic resonance imaging (fMRI) scan. The resting-state function connectivity between the DMN and CEN was analyzed, with the seed of medial prefrontal cortex (MPFC) and dorsolateral PFC (DLPFC), respectively. The results were also compared with 33 healthy controls.

Results: With the mPFC seed, the remitted depression showed a lower DMN connectivity in frontal, temporal, and parietal cortexes, compared with the other two groups. With the DLPFC seed, the current and remitted depression shared similar pattern (lower connectivity) in frontal cortex, but differed in parietal cortex (lower connectivity in remitted patients), within the CEN as well as between the DLPFC seed and DMN.

Conclusions: The lower DMN function connectivity in frontal, temporal, and parietal cortexes in remitted depression suggests a potential over-compensation (inhibition) mechanism behind the remission of depression. The different connectivity model in parietal cortex between the current and remitted depression emphasizes the parietal cortex in the remission of depression, which might be a reliable biomarker of remission. The similar connectivity pattern in the frontal cortex between two patient groups hints a trait-like neural basis of depressive episode, which might be a stable predictor of recurrence.

Significance: The present study offers a first experimental evidence of function connectivity between DMN and CEN in the current and remitted depression, which helps to uncover the neural mechanism during the remission of depression.

\section{Keywords}

Depression, Default mode network (DMN), Central executive network (CEN), Remitted depression (RMD), FMRI

\footnotetext{
'Psychology \& Social College, Shenzhen University, Shenzhen, China

2Department of Psychology, the Third Military Medical University, Chong Qing, China

${ }^{3}$ Department of Nursing, the Third Military Medical University, Chong Qing, China

${ }^{4}$ Radiological Department, the Southwest Hospital of the Third Military Medical University, Chong Qing, China

${ }^{5}$ Data Center of Affiliated hospital of Zhejiang University, Hang Zhou, China

${ }^{\dagger}$ Author for correspondence: Zhengzhi Feng, Department of psychology, The Third Military Medical University, Chong qing, 400038, China, Tel: 86-023-68771591; Fax: +86-023-68771591; email: fzz@tmmu.edu.cn
} 


\section{Introduction}

Depression is one of the most commonly occurred mental disorders [1]. Patients with major depressive disorder (MDD) have suffered from cognitive disturbance [2,3], which is closely correlated with rumination [4-6]. The default mode network (DMN) has been indicated involving in rumination, self-referent cognition, and memory retrieval, which includes areas of medial prefrontal cortex (mPFC), posterior cingulated cortex, and multiple (mainly medial) parts of posterior parietal cortex [7-9]. Abnormal functional alterations of the DMN have been confirmed in the firstepisode, drug-naïve major depressive disorder patients [10]. A large area of the DMN in depression has shown a decrease in coherence to the network as a whole, which is enhanced after electroconvulsive therapy (ECT) in those improving clinically, but remains unchanging in patients not responding to ECT [11]. The central executive network (CEN), in contrast, plays a critical role in regulating attention, working memory, and decision making [12], which includes dorsolateral prefrontal cortex (DLPFC) and multiple (mainly lateral) parts of posterior parietal cortex. The disturbed CEN activity has been reported relating to cognitive disturbance in depression [13]. Collectively, the DMN might be a neural mechanism of depressive rumination, while the CEN might be a neural basis of cognitive disturbance in depression. However, the relationship between DMN and CEN in depression has seldom been addressed.

In general population, the activation of the DLPFC by TMS stimulation modulated DLPFC (a part of CEN) - DMN connectivity, which suggested a connection between DMN and CEN [14]. The resting-state time course of the defaultmode network was negatively correlated with that of the "task-positive network", a collection of regions commonly recruited in demanding cognitive tasks, which is much like the CEN $[15,16]$. In depressed patients, recent study observed and confirmed that the functional connectivity was abnormally elevated within the DMN and diminished within the CEN, and connectivity between these two networks was altered [17]. Similarly, for individuals at high risk for depression, it was found that they had increased DMN connectivity, as well as reduced inverse connectivity between the DMN and CEN [18]. These results highlight a connection between DMN and CEN in depression.
Constantly, the mPFC has been reported as a part of DMN [7-9], while the DLPFC has been included as a part of CEN [12]. Specifically, DLPFC was mostly ascribed "cognitive" or "executive" functions, whereas mPFC was primarily associated with "emotional" or "affective" functions [19]. Independently, it has been reported that $\mathrm{mPFC}$ reactivity predicted rumination [20], related to selfreferential events [21], as a part of DMN [22], while DLPFC has been known to be activated as cognitive hierarchy increases [23]. Consistently, it has been indicated that the $\mathrm{MPFC}$ and DLPFC was highly interacted [19,24]. However, the interaction between mPFC seeded DMN and DLPFC seeded CEN in depression remained unclear.

Moreover, results of altered function connectivity in DMN were mainly obtained from the current depression $[10,11,17,18]$, parts of them came from the remitted depression [25-27]. Among which, it was reported that the disrupted topological organization of the DMN might be considered as a potential biomarker of the episodic memory deficits in remitted late-onset depression patients [28]. For CEN, limited results confirmed the diminished connectivity in depression [14,15,17]. Importantly, no evidence of CEN connectivity was reported in remitted depression. Thus, whether the altered connectivity within CEN, as well as between $\mathrm{DMN}$ and CEN, play a role in the remission of depression, remains unclear.

In conclusion, the present study selected the mPFC as seed of DMN and DLPFC as seed of CEN, to observe the network connectivity between DMN and CEN in the current and remitted depression. Our hypotheses were: First, the patients with current depression possessed altered function connectivity within DMN and CEN, as well as between DMN and CEN. Second, the individuals with current and remitted depression would share similarity and dissimilarity in function connectivity within DMN and CEN, as well as between DMN and CEN.

\section{Methods \\ - Participants}

Three groups of participants were recruited: patients with current depression, individuals with remitted depression (RMD), and never depressed healthy controls (NC). The normal controls 
were recruited through advertisement, and the depressed individuals were obtained through both advertisement and clinical psychiatrists, from psychiatric hospital of Chongqing, China. The depressive symptoms were assessed by the Patient Health Questionnaire (PHQ-9) [29], the Beck Depression Inventory-II (BDI-II) [30], the Hamilton Depression Rating Scale (HDRS, 24 items) [31], and the Diagnostic and Statistical Manual of Mental Disorders-V (DSM-V) [32]. To observe the anxiety level of subjects, the Beck Anxiety Inventory-II (BAI) was further investigated. The criteria for each group were as following: patients with current depression should be diagnosed as MDD by clinical psychiatrists based on the DSM-V, with two or more episodes [7,33]; the individuals of remitted depression should be remitted from last episode for at least 6 months and free from antidepressants at least 3 months; while the healthy controls should be exempted from the current or lifetime depression or psychosis. Exclusion criterion: Infection, trauma, and allergy within two weeks, bipolar disorder, schizophrenia, severe physiological disease, learning disability, drug dependence, history of psychosis, history of alcohol or drug abuse, and structural brain lesions. For the security of functional magnetic resonance imaging (fMRI) scan, participants with the following circumstance were also excluded: Any metal or magnetic materials within the body, having a pacemaker or being pregnant, a history of neurosurgery or epilepsy. The detailed recruiting procedure could be found in Figure 1 and our previous work [34]. Originally, 156 participants (healthy and patients) were invited, 105 of whom agreed to participate, after excluding unexpected situations such as no show of participants, scan machine unavailable, claustrophobia, unexpected discharge, brain lesions, or excessive head motion, 33 healthy controls, 30 MDD patients, and 27 remitted individuals were obtained finally. All MDD patients were medicated (ten were taking mirtazapine, 30 - $40 \mathrm{mg}$; eight were taking fluoxetine hydrochloride, 20 - $40 \mathrm{mg}$; twelve were taking paroxetine hydrochloride, $20-30$ $\mathrm{mg})$.

The research protocol was approved by the Human Research Ethics Committee of the Third Military Medical University. Candidate participants (patients and healthy controls) were interviewed through telephone by a trained doctoral - level psychological personnel. For qualified subjects, the experiment was dated through telephone. After the subjects' arrival, the details of the experiment were informed again, and the voluntaries to participate in the experiment and the right to quit during the experiment were emphasized. After written informed consents were signed, a 10 minutes rest was set before the experiment to allow the

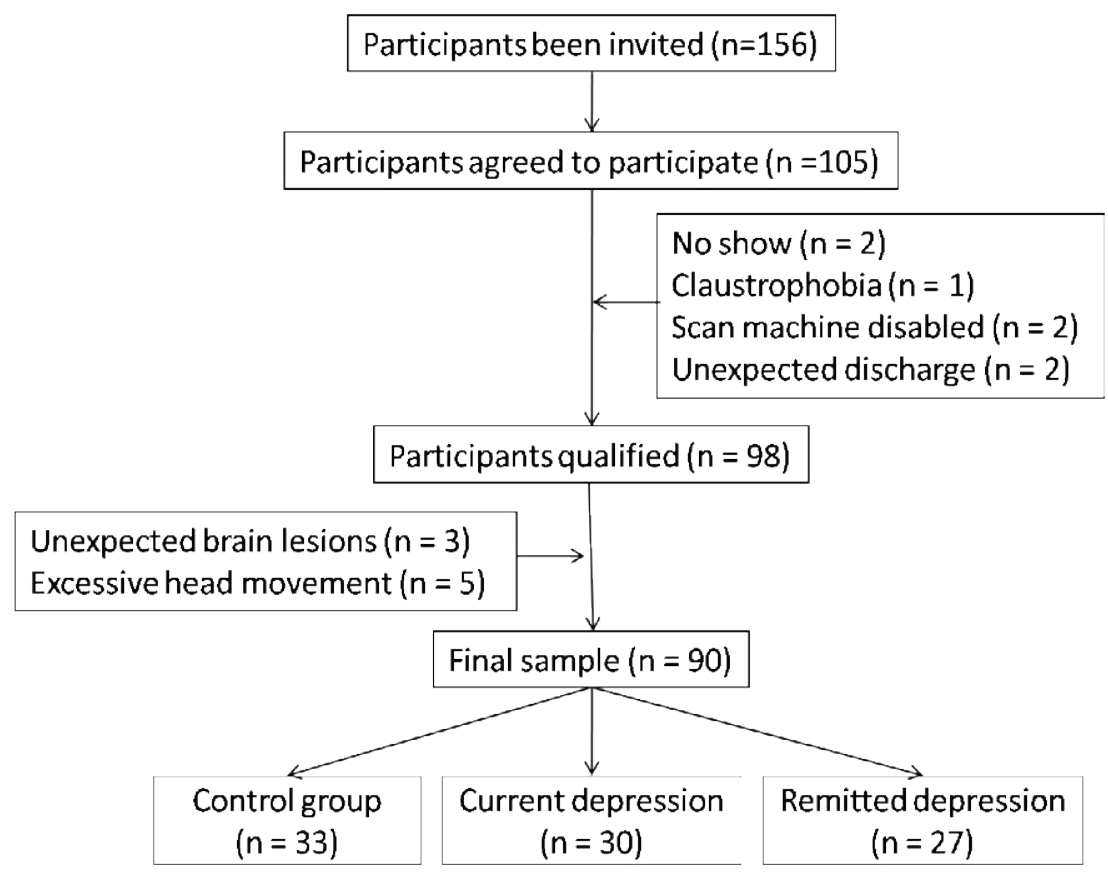

Figure 1: Flow chart of participant recruitment. 
familiarity with the environment.

\section{- Instrument}

The BDI-II and PHQ were used to assess depression level. Specifically, BDI-II reflected the depression severity with 21 self-report items [30,35], while the PHQ mainly examined the frequency of depressive symptoms referring to 9 diagnostic criterion of MDD [36]. The BAI was used to examine the anxiety level, which comprised of 21 self-report items too [35,37].

\section{- fMRI data acquisition}

Functional images were acquired on a 3.0-T Siemens TimTrio whole-body MRI system (Siemens Medical Solutions, Erlangen, Germany) with a 12-channel phased-array head coil, located at Southwest Hospital, Chongqing, China. Image data were obtained using single shot T2-weighted EPI (echo planar imaging, TR/ $\mathrm{TE}=2000 / 30 \mathrm{~ms}$, flip angle $=90^{\circ}$, reconstruction matrix $=64 \times 64$, field of view $(F O V)=384 \mathrm{~mm}$, $4 \mathrm{~mm}$ slice thickness with $1 \mathrm{~mm}$ gap, voxel size $=3.0 \mathrm{~mm} \times 3.0 \mathrm{~mm} \times 3.0 \mathrm{~mm}$, number of slices per volume=36). For each subject, a total of 240 volumes were acquired, resulting in a total scan time of $480 \mathrm{~s}$. The anatomical images were acquired through 3D-T1MPRAGE protocol (inversion time (TI) / repetition time (TR) / echo time $(\mathrm{TE})=900 / 2530 / 2.34 \mathrm{~ms}$, flip angle $=7 \circ$, reconstruction matrix $=256 \times 256, \mathrm{FOV}=256 \mathrm{~mm}$, slice thickness=1 mm, number of slices=192).

\section{- fMRI data preprocessing}

The resting-state function data was preprocessed through the Statistical Parametric Mapping package (SPM8, http://www.fil.ion.ucl.ac.uk/ spm/software/spm8/), which was analyzed after removing the first 10 scan images. The basic procedures were: (a) slice timing, (b) realign, (c) coregister, (d) segment, (e) normalize (by DARTEL, with re-sampling rate $3 \mathrm{~mm} \times 3 \mathrm{~mm}$ $\times 3 \mathrm{~mm}(1 \mathrm{~mm} \times 1 \mathrm{~mm} \times 1 \mathrm{~mm}$ for anatomic data)); (f) smooth (FWHM=6mm). During the preprocessing, the head motion, white matter, and cerebrospinal fluid, were regressed out.

\section{- Statistics}

Function connectivity was analyzed with REST 1.8 (Resting-State fMRI Data Analysis Toolkit) [38]. The MNI coordinate was consistent with the literature, $\operatorname{mPFC}(-4,48,-15)$ [39)], DLPFC [40-42,33]. The DMN connectivity was obtained by the whole-brain function connection with the seed of $\mathrm{mPFC}$, while the CEN connectivity template was generated from the previous findings [12]. The within network function connection was obtained with seed of $\mathrm{mPFC}(\mathrm{DMN})$ and DLPFC (CEN) respectively, while the between network function connection was obtained between DLPFC seed and DMN as well as between mPFC seed and CEN. See Supplementary materials (Figures S1 and S2) for details.

After individual analysis, an one-way ANOVA was conducted, and a further two-sample t-test was applied to compare each of two groups (AlphaSim correction, $\mathrm{p}<0.01$ (uncorrected p-value $<0.001)$, voxel threshold $>40$, size of kernel smoothing $=6 \mathrm{~mm}$ (Full width at half maximum, FWHM), whole-brain mask). Gender and age were included as co-variables. A correlation analysis was also conducted between functional connectivity and the clinical variables (duration of illness, number of episodes, and scores of BDI, BAI, PHQ, HDRS) of depressed patients with REST 1.8. The final image was overlaid on a ch2 bet.nii template, and figured with Rest Viewer [38]. The basic information of participants was analyzed through $\chi^{2}$ test and one-way ANOVA.

\section{Results \\ - Group information (Table 1)}

Three groups did not differ on the gender ratio $\left(\chi^{2}=1.792, \mathrm{df}=2, \mathrm{~N}=89, \mathrm{p}=0.408\right)$, age $(\mathrm{F}$

\begin{tabular}{|c|c|c|c|c|c|c|c|c|c|c|}
\hline & $\begin{array}{l}\text { Gender } \\
\text { (M/F) }\end{array}$ & Age & $\begin{array}{l}\text { Education } \\
\text { level }\end{array}$ & Episodes & $\begin{array}{l}\text { Duration } \\
\text { of illness } \\
\text { (year) }\end{array}$ & $\begin{array}{l}\text { FD value } \\
\text { of head } \\
\text { motion }\end{array}$ & PHQ & BDI & HDRS & BAI \\
\hline$N C(n=33)$ & $12 / 21$ & $48.85 \pm 12.86$ & $2.33 \pm .74$ & & & $0.13 \pm 0.06$ & $1.09 \pm 1.26$ & $2.12 \pm 1.62$ & $1.76 \pm 1.12$ & $1.76 \pm 3.24$ \\
\hline $\operatorname{MDD}(n=30)$ & $7 / 23$ & $48.77 \pm 11.88$ & $2.17 \pm 0.70$ & $3.1 \pm 1.37$ & $8.75 \pm 6.94$ & $0.11 \pm 0.06$ & $21.00 \pm 5.80$ & $35.86 \pm 10.12$ & $23.43 \pm 2.94$ & $11.89 \pm 6.86$ \\
\hline $\operatorname{RMD}(n=27)$ & $10 / 16$ & $49.56 \pm 10.77$ & $2.1 \pm 0.80$ & $2.56 \pm 1.39$ & $9.76 \pm 5.16$ & $0.15 \pm 0.12$ & $5.41 \pm 3.88$ & $6.70 \pm 4.51$ & $5.11 \pm 1.91$ & $4.93 \pm 5.02$ \\
\hline$p$ & 0.408 & 0.963 & 0.479 & 0.199 & 0.578 & 0.127 & $<0.001$ & $<0.001$ & $<0.001$ & $<0.001$ \\
\hline \multicolumn{11}{|c|}{$\begin{array}{l}\text { Note: } \mathrm{M}=\text { male. } \mathrm{F}=\text { female. } \mathrm{BAI}=\text { Beck Anxiety Inventory. } \mathrm{BDI}=\text { Beck Depression Inventory. } \mathrm{PHQ}=\text { patient health questionnaire. } \mathrm{HDRS}=\text { the Hamilton } \\
\text { Depression Rating Scale. Education level: } 1=\text { primary school or lower, } 2=\text { middle school, } 3=\text { university, } 4=\text { postgraduates. } \mathrm{NC}=\mathrm{Never} \text { disordered healthy } \\
\text { Controls. } \mathrm{MDD}=\text { Major Depressive Disorder. RMD = Remitted depression. }\end{array}$} \\
\hline
\end{tabular}


$(2,87)=0.037, \quad \mathrm{p}=0.963, \quad$ partial-eta $\left.{ }^{2}=0.002\right)$, or education level $(\mathrm{F}(2,87)=0.743, \mathrm{p}=0.479$, partial-eta $\left.{ }^{2}=0.017\right)$. As expected, they differed on the scores of PHQ $(\mathrm{F}(2,87)=210.829, \mathrm{p}<0.001$, partial-eta $\left.{ }^{2}=0.835\right)$, BDI $(\mathrm{F} \quad(2,87)=254.812$, $\mathrm{p}<0.001, \quad$ partial-eta $\left.{ }^{2}=0.845\right), \quad$ BAI $\quad(\mathrm{F}$ $(2,87)=29.674, \mathrm{p}<0.001$, partial-eta $\left.{ }^{2}=0.41\right)$, and HDRS (F $(2,87)=935.236, \mathrm{p}<0.001$, partialeta $\left.{ }^{2}=0.95\right)$, between each of two groups, with higher scores in MDD group and lower scores in NC group. Three groups did not differ in head motion $(\mathrm{F}(2,87)=2.114, \mathrm{p}=0.127$, partialeta $2=0.10$ ). The current and remitted depression did not differ on the numbers of episodes and duration of illness $(\mathrm{p}>0.05)$.

\section{- Function connectivity within the DMN and CEN}

The resting-state function connectivity within the DMN (mPFC seed) was compared with one-way ANOVA between three groups, which indicated that three groups differed between mPFC seed and left inferior temporal cortex $(\mathrm{k}=65, \mathrm{p}<0.01)$, left $(\mathrm{k}=57, \mathrm{p}<0.01)$ and right precuneus $(\mathrm{k}=14, \mathrm{p}<0.01)$, left parietal cortex $(\mathrm{k}=77, \mathrm{p}<0.01)$, left middle cingulum $(\mathrm{k}=57$, $\mathrm{p}<0.01)$, and left middle frontal cortex $(\mathrm{k}=38$, $\mathrm{p}<0.01)$. Further two-sample t-test indicated that patients with MDD had lower connectivity between $\mathrm{mPFC}$ seed and right cerebrum $(\mathrm{k}=93$, $\mathrm{p}<0.01)$, left inferior orbitofrontal cortex $(\mathrm{k}=22$, $\mathrm{p}<0.01)$, as well as left middle cingulum $(\mathrm{k}=41$, $\mathrm{p}<0.01$ ), compared with NC. Remitted patients, surprisingly, showed lowest connection between mPFC seed and left superior frontal cortex $(\mathrm{k}=42, \mathrm{p}<0.01)$, left middle temporal cortex $(\mathrm{k}=68, \mathrm{p}<0.01)$, left posterior cingulate $(\mathrm{k}=81$, $\mathrm{p}<0.01)$, as well as right precuneus $(\mathrm{k}=48$, $\mathrm{p}<0.01$ ), compared with the other two groups Figures 2 and 3. See more details in Table 2 and Supplementary materials (Figure S3) for details.

The function connectivity within the CEN (DLPFC seed) was compared with one-way ANOVA between three groups, which indicated that three groups differed between DLPFC seed and left $(\mathrm{k}=43, \mathrm{p}<0.01)$ and right inferior parietal cortex $(\mathrm{k}=72, \mathrm{p}<0.01)$. Further two-sample t-test indicated that patients with MDD had lower connectivity between DLPFC seed and right middle frontal cortex $(\mathrm{k}=21, \mathrm{p}<0.01)$, compared with normal controls. Remitted patients, in contrast, they had lower connectivity between DLPFC seed and right middle frontal cortex $(\mathrm{k}=39, \mathrm{p}<0.01)$, particular the right middle orbitofrontal cortex $(\mathrm{k}=24, \mathrm{p}<0.01)$, compared with the normal controls. However, they differed in connectivity (lower connectivity) with MDD in function connectivity between DLPFC seed and right superior parietal cortex $(\mathrm{k}=18, \mathrm{p}<0.01)$, as well as left inferior parietal lobule $(\mathrm{k}=44, \mathrm{p}<0.01)$. See more details in Table 3 and Supplementary materials (Figure S4).

\section{- Function connectivity between the DMN and CEN}

The function connectivity between DLPFC seed (CEN) and DMN was compared with one-way ANOVA between three groups, which indicated that three groups differed between DLPFC seed and left $(\mathrm{k}=44, \mathrm{p}<0.01)$ and right inferior parietal cortex $(\mathrm{k}=11, \mathrm{p}<0.01)$, right middle temporal cortex $(\mathrm{k}=48, \mathrm{p}<0.01)$, and right inferior orbitofrontal cortex $(\mathrm{k}=35$, $\mathrm{p}<0.01)$. Further two-sample t-test indicated a group difference only between DLPFC seed and DMN. Patients with MDD showed lower connectivity between DLPFC seed and left medial orbitofrontal cortex $(\mathrm{k}=40, \mathrm{p}<0.01)$, right superior medial frontal cortex $(\mathrm{k}=103, \mathrm{p}<0.01)$, as well as right superior temporal cortex $(k=69$, $\mathrm{p}<0.01$ ), compared with the normal controls. The remitted patients showed lower connection of connectivity between DLPFC seed and right inferior orbitofrontal cortex $(\mathrm{k}=45, \mathrm{p}<0.01)$, left middle cingulum $(\mathrm{k}=35, \mathrm{p}<0.01)$, compared with the normal controls. However, they differed in connectivity (lower connectivity) with MDD in parietal function connectivity, with lower connectivity between DLPFC seed and right inferior parietal lobule $(\mathrm{k}=55, \mathrm{p}<0.01)$ as well as left inferior parietal lobule $(\mathrm{k}=38, \mathrm{p}<0.01)$ (Figure 4 and Table 4).

\section{- Correlation between functional connectivity and the clinical variables of patients}

Correlation between functional connectivity and the clinical variables (duration of illness, number of episodes, and scores of BDI, BAI, PHQ, HDRS) of patients was conducted with REST 1.8, which suggested a negative correlation in MDD patients, between DLPFC-DMN functional connectivity and duration of illness $(\mathrm{r}=-0.73, \mathrm{k}=51, \mathrm{p}<0.01$, in left parietal cortex), as well as number of episodes $(r=-0.80, k=87$, $\mathrm{p}<0.01$, in right superior medial frontal cortex), while a positive correlation was found between mPFC-DMN functional connectivity and BDI scores $(\mathrm{r}=0.78, \mathrm{k}=39, \mathrm{p}<0.01$, in left medial frontal cortex). RMD patients, in contrast, a negative correlation was only reported between 
Research Qin Dai
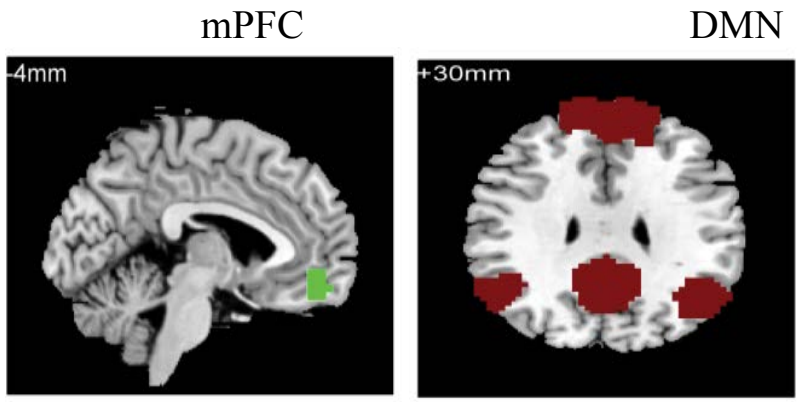

A MDD-NC
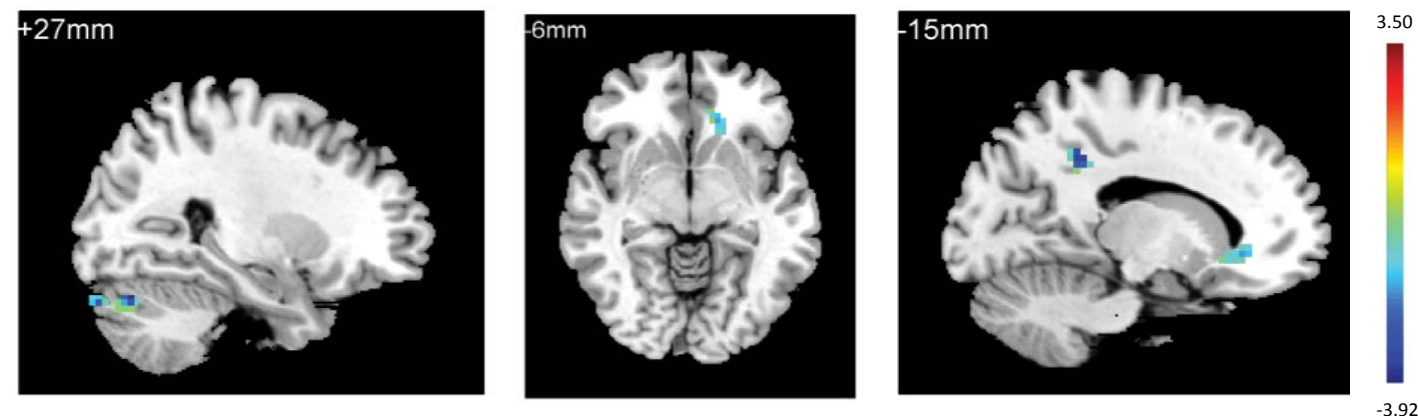

Cerebelum R

Frontal_Inf_Orb L

Cingulum_Mid L

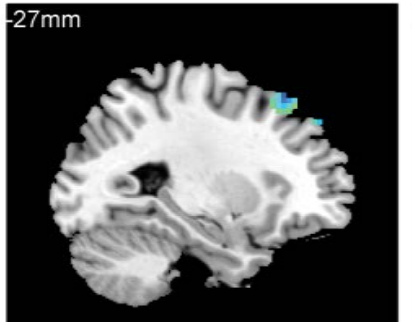

Frontal Mid L

Parietal Lobe L

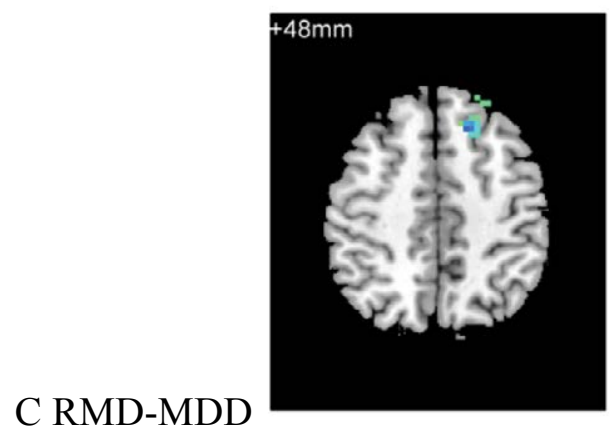

Frontal_Sup L
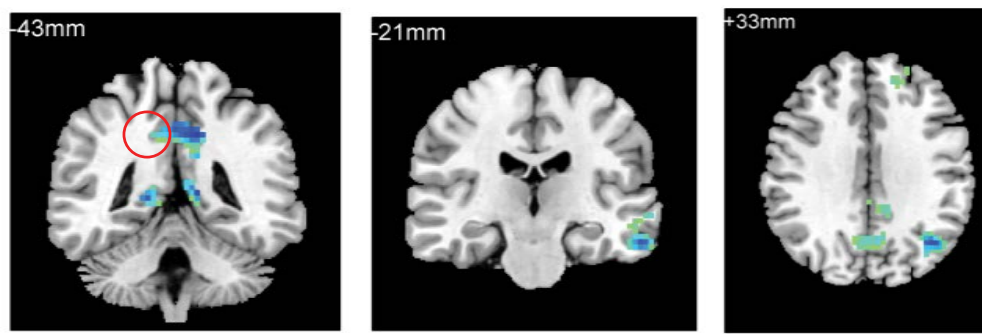

$-5.59$

RMD-NC

Cingulum Mid L

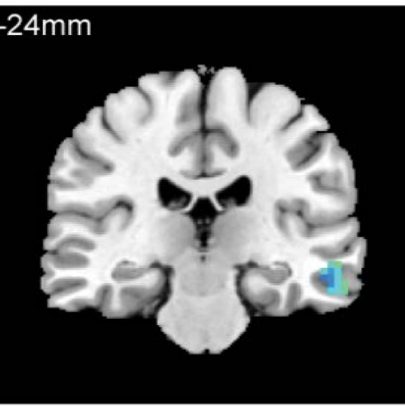

Temporal_Mid L
Temporal Mid L

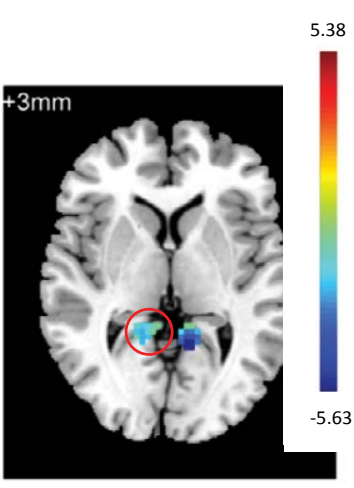

Posterior Cingulate and

precuneus

Figure 2: Function connectivity within the DMN (mPFC seed). 
Default Model Network Mechanism in Current and Remitted Depression Research
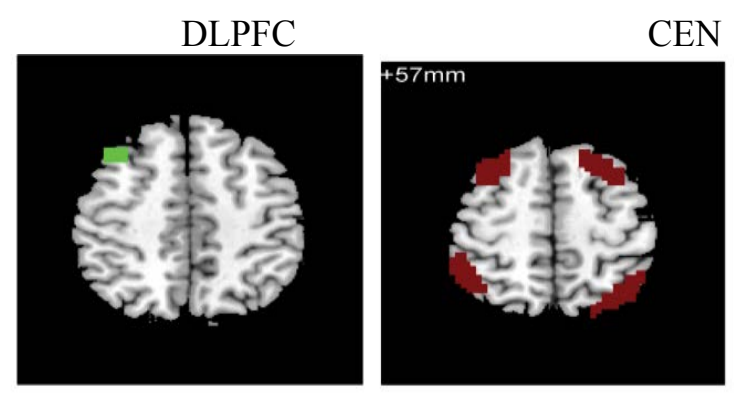

A MDD-NC

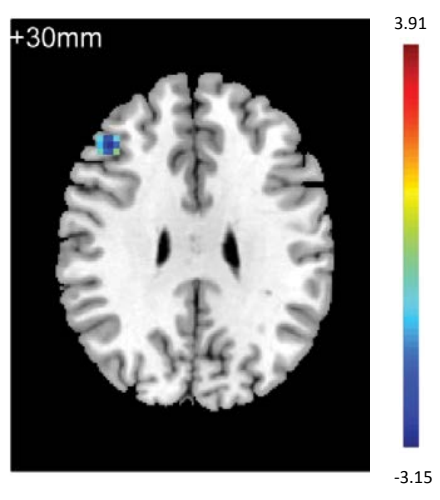

Frontal_Mid R

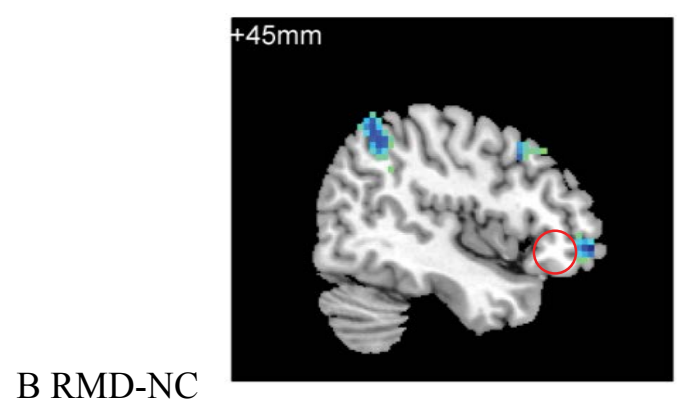

Frontal_Mid_Orb R

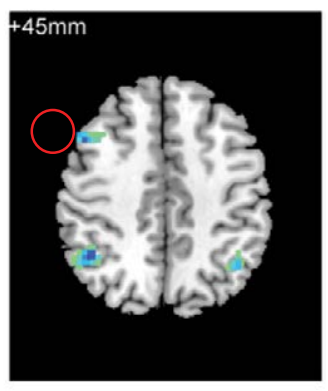

Frontal_Mid R

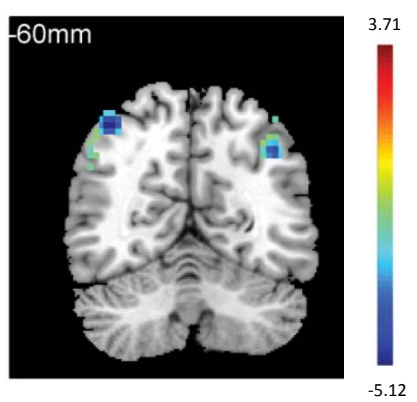

Parietal

Lobe

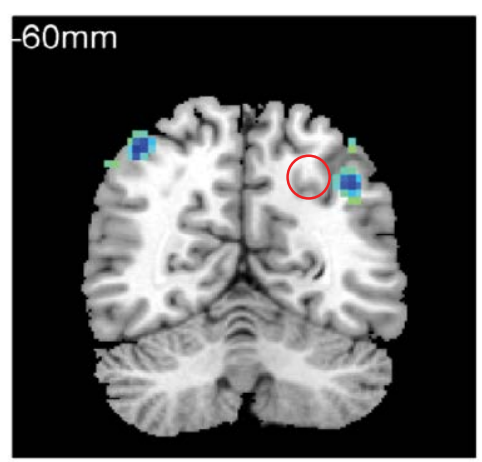

Parietal Lobule L

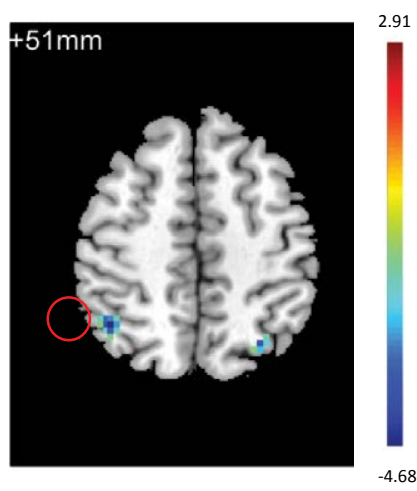

Parietal Lobule R

Figure 3: Function connectivity within the CEN (DLPFC seed). 
Research Qin Dai

\begin{tabular}{|c|c|c|c|c|c|c|c|}
\hline \multirow[t]{2}{*}{ mPFC-DMN } & \multirow[t]{2}{*}{ Cortex } & \multirow[t]{2}{*}{ Lateral } & \multirow[t]{2}{*}{ F/t value } & \multirow[t]{2}{*}{ Cluster (k) } & \multicolumn{3}{|c|}{ MNI coordinate } \\
\hline & & & & & $\mathrm{x}$ & $y$ & $z$ \\
\hline \multirow{6}{*}{$\begin{array}{l}\text { ANOVA of three } \\
\text { groups }\end{array}$} & Temporal_Inf & L & 11.44 & 65 & -57 & -24 & -21 \\
\hline & Pracuneus & $R$ & 9.70 & 14 & 18 & -45 & 3 \\
\hline & Pracuneus & L & 16.26 & 57 & -12 & -48 & 9 \\
\hline & Parietal Lobe & L & 11.55 & 77 & -39 & -66 & 33 \\
\hline & Cingulum_Mid & L & 15.56 & 57 & 0 & -39 & 42 \\
\hline & Frontal_Mid & $\mathrm{L}$ & 9.42 & 38 & -27 & 27 & 57 \\
\hline \multirow[t]{3}{*}{ MDD-NC } & Cerebrum & $\mathrm{R}$ & -3.74 & 93 & 27 & -75 & -27 \\
\hline & Frontal_Inf_Orb & L & -2.86 & 22 & -15 & 30 & -6 \\
\hline & Cingulum_Mid & $\mathrm{L}$ & -3.68 & 41 & -15 & -42 & 39 \\
\hline \multirow[t]{6}{*}{ RMD-NC } & Frontal_Mid & L & -4.34 & 42 & -27 & 27 & 57 \\
\hline & Cingulum_Mid & L & -4.67 & 20 & -9 & -43 & 41 \\
\hline & Temporal_Mid & L & -4.54 & 77 & -60 & -21 & -21 \\
\hline & Pracuneus & $\mathrm{R}$ & -4.44 & 19 & 15 & -42 & 3 \\
\hline & Pracuneus & L & -5.59 & 186 & -12 & -48 & 9 \\
\hline & Parietal Lobe & L & -4.70 & 80 & -42 & -66 & 33 \\
\hline \multirow[t]{4}{*}{ RMD-MDD } & Frontal_Sup & $\mathrm{L}$ & -4.41 & 42 & -15 & 27 & 48 \\
\hline & Temporal_Mid & $\mathrm{L}$ & -4.23 & 68 & -54 & -24 & -15 \\
\hline & Posterior Cingulate & L & -5.63 & 81 & -12 & -54 & 3 \\
\hline & Pracuneus & $\mathrm{R}$ & -3.95 & 48 & 15 & -48 & 3 \\
\hline
\end{tabular}

\section{Table 3: Function connectivity within CEN (DLPFC seed).}

\begin{tabular}{|c|c|c|c|c|c|c|c|}
\hline \multirow[t]{2}{*}{ DLPFC-CEN } & \multirow[t]{2}{*}{ Cortex } & \multirow[t]{2}{*}{ Lateral } & \multirow[t]{2}{*}{ F/t value } & \multirow[t]{2}{*}{ Cluster (k) } & \multicolumn{3}{|c|}{ MNI coordinate } \\
\hline & & & & & $\mathbf{x}$ & $\mathbf{y}$ & $\mathbf{z}$ \\
\hline \multirow{2}{*}{$\begin{array}{l}\text { ANOVA of three } \\
\text { groups }\end{array}$} & Inferior Parietal Lobule & $\mathrm{L}$ & 12.01 & 43 & -42 & -60 & 42 \\
\hline & Inferior Parietal Lobule & $\mathrm{R}$ & 15.40 & 72 & 42 & -57 & 51 \\
\hline MDD-NC & Frontal_Mid & $\mathrm{R}$ & -3.07 & 21 & 40 & 30 & 30 \\
\hline \multirow[t]{4}{*}{ RMD-NC } & Frontal_Mid_Orb & $\mathrm{R}$ & -5.07 & 24 & 45 & 51 & -6 \\
\hline & Frontal_Mid & $\mathrm{R}$ & -4.09 & 39 & 48 & 15 & 45 \\
\hline & Parietal_Sup & $\mathrm{R}$ & -5.12 & 18 & 39 & -60 & 54 \\
\hline & Inferior Parietal Lobule & $\mathrm{L}$ & -4.64 & 44 & -42 & -60 & 42 \\
\hline \multirow[t]{2}{*}{ RMD-MDD } & Inferior Parietal Lobule & $\mathrm{L}$ & -4.35 & 47 & -42 & -60 & 39 \\
\hline & Inferior Parietal Lobule & $\mathrm{R}$ & -4.69 & 48 & 42 & -57 & 51 \\
\hline
\end{tabular}

DLPFC-DMN functional connectivity and duration of illness, in right superior orbitofrontal cortex $(\mathrm{r}=-0.85, \mathrm{k}=32, \mathrm{p}<0.01)$, right medial frontal cortex $(\mathrm{r}=-0.91, \mathrm{k}=47, \mathrm{p}<0.01)$, left middle temporal cortex $(\mathrm{r}=-0.92, \mathrm{k}=65, \mathrm{p}<0.01)$, and left middle cingulate cortex (in left inferior parietal cortex, $\mathrm{r}=0.89, \mathrm{k}=113, \mathrm{p}<0.01$; right inferior parietal cortex, $\mathrm{r}=0.81, \mathrm{k}=92, \mathrm{p}<$ 0.01 ; and right middle frontal cortex, $r=0.72$, $\mathrm{k}=53, \mathrm{p}<0.01$ ), between number of episodes and DLPFC-DMN functional connectivity (in left medial frontal cortex, $\mathrm{r}=0.84, \mathrm{k}=30, \mathrm{p}<$ 0.01 ; and left superior frontal cortex, $\mathrm{r}=0.79, \mathrm{k}$ $=35, \mathrm{p}<0.01$ ), and between number of episodes and $\mathrm{mPFC}-\mathrm{DMN}$ functional connectivity (in right superior medial frontal cortex, $\mathrm{r}=0.66, \mathrm{k}=$ $41, \mathrm{p}<0.01)$, as well as between mPFC-DMN functional connectivity and BDI scores (in left parietal cortex, $r=0.73, k=28, p<0.01)$. See Figure 55 of Supplemental materials for details.

\section{Discussion}

The present study offered a first experimental evidence of function connectivity interaction between DMN and CEN in both current and remitted depression. The current and remitted depression shared similar pattern in frontal cortex connectivity, but differed in parietal cortex connectivity (DLPFC seed, between DMN and CEN as well as within CEN), which suggested different roles of two cortexes in the remission of depression. The remitted depression also showed lower connectivity in frontal, temporal, and parietal cortexes (mPFC seed, within DMN), which indicated a potential over-compensation (inhibition) mechanism in the remission process 
Default Model Network Mechanism in Current and Remitted Depression Research

DLPFC
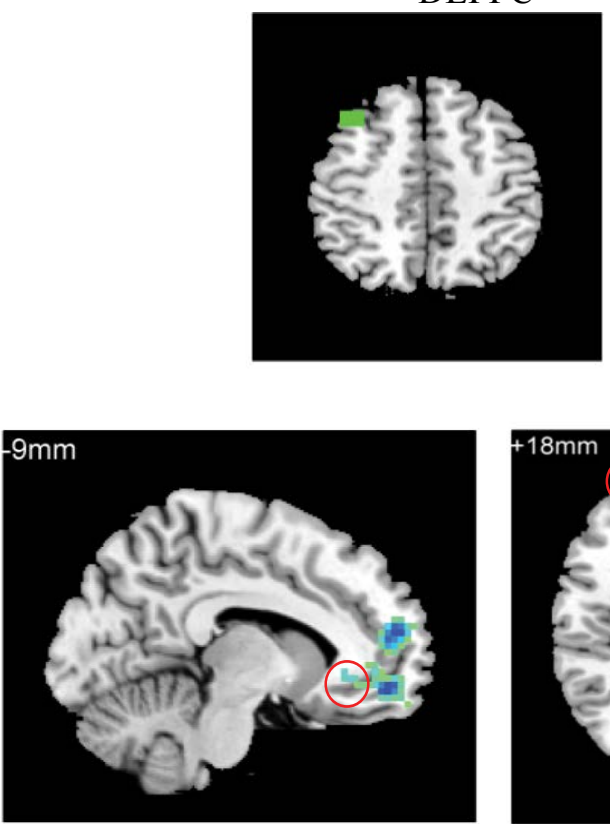

A MDD-NC

Frontal_Med_Orb L
DMN
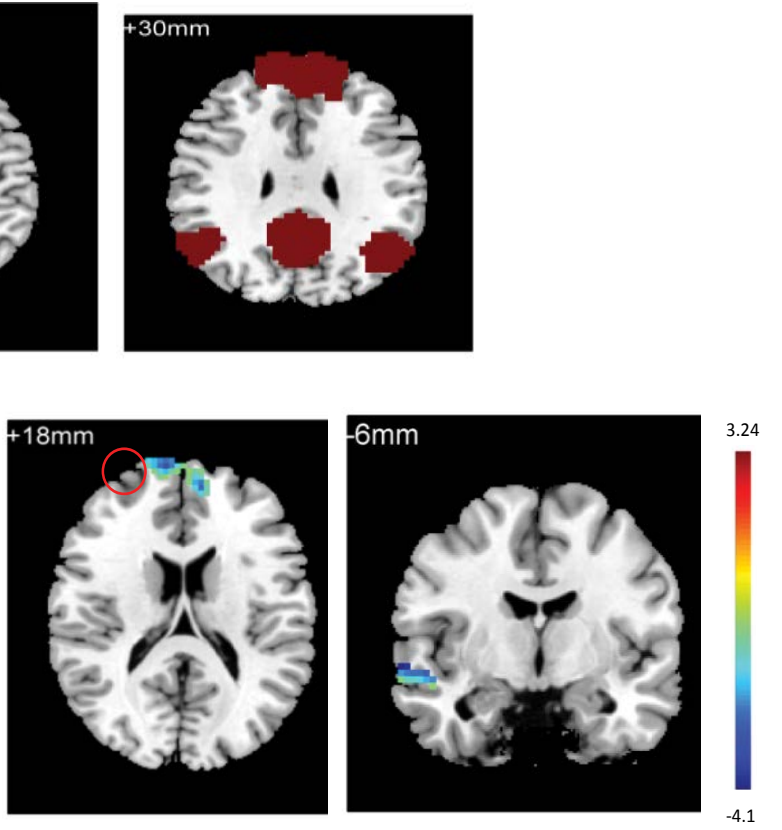

Frontal_Sup_Medial R

Temporal_Sup R
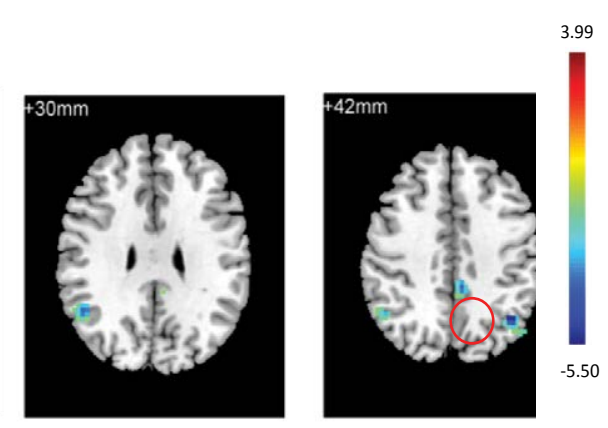

B RMD-NC

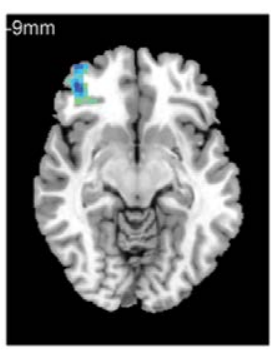

Frontal_Inf_Orb R Inferior Parietal L

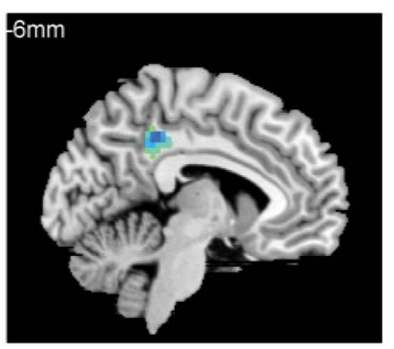

Cingulum_Mid L
Inferior Parietal R 
Research Qin Dai

\begin{tabular}{|c|c|c|c|c|c|c|c|}
\hline \multirow[t]{2}{*}{ DLPFC-DMN } & \multirow[t]{2}{*}{ Cortex } & \multirow[t]{2}{*}{ Lateral } & \multirow[t]{2}{*}{ F/t value } & \multirow[t]{2}{*}{ Cluster (k) } & \multicolumn{3}{|c|}{ MNI coordinate } \\
\hline & & & & & $\mathbf{x}$ & $\mathbf{y}$ & $\mathbf{z}$ \\
\hline \multirow{4}{*}{$\begin{array}{l}\text { ANOVA of three } \\
\text { groups }\end{array}$} & Temporal_Mid & $\mathrm{R}$ & 11.06 & 48 & 66 & -27 & -9 \\
\hline & Frontal_Inf_Orb & $\mathrm{R}$ & 9.72 & 35 & 42 & 42 & -9 \\
\hline & Inferior Parietal Lobule & $\mathrm{R}$ & 10.24 & 11 & 51 & -54 & 30 \\
\hline & Inferior Parietal Lobule & $\mathrm{L}$ & 17.01 & 44 & -42 & -60 & 42 \\
\hline \multirow[t]{3}{*}{ MDD-NC } & Frontal_Med_Orb & $\mathrm{L}$ & -3.57 & 40 & -9 & 51 & -12 \\
\hline & Frontal_Sup_Medial & $\mathrm{R}$ & -3.50 & 103 & 9 & 66 & 18 \\
\hline & Temporal_Sup & $\mathrm{R}$ & -4.10 & 69 & 66 & -6 & -6 \\
\hline \multirow[t]{4}{*}{ RMD-NC } & Frontal_Inf_Orb & $\mathrm{R}$ & -4.64 & 45 & 42 & 54 & -9 \\
\hline & Cingulum_Mid & $\mathrm{L}$ & -4.35 & 35 & -6 & -39 & 42 \\
\hline & Inferior Parietal Lobule & $\mathrm{R}$ & -4.34 & 55 & 51 & -54 & 30 \\
\hline & Inferior Parietal Lobule & $\mathrm{L}$ & -5.50 & 38 & -42 & -60 & 42 \\
\hline RMD-MDD & Inferior Parietal Lobule & $\mathrm{L}$ & -5.24 & 47 & -42 & -60 & 42 \\
\hline
\end{tabular}

of depression. Our findings helped to uncover the neural mechanism during the remission of depression.

The most important improvement of the current work over literature was to observe the connectivity between DMN and CEN in remitted patients. Within the DMN (mPFC seed), the remitted patients showed the lowest function connectivity in frontal, temporal, and parietal cortexes, compared with the other two groups. That is to say, the improvement of clinical depression is achieved by over suppressing the function connectivity within the DMN. Inspired by the literature, which indicated that nonresponders to dorsomedial prefrontal cortex TMS treatment showed significantly lower connectivity through a classical reward pathway comprising ventral tegmental area, striatum, and a region in ventromedial prefrontal cortex (mPFC) [37]. In those improving clinically, in contrast, the decreased network coherence in DMN was normalized after electroconvulsive therapy (ECT) [11]. Taken together, for remitted depression, a similar connectivity model with the healthy controls in the DMN (mPFC seed) could be expected, which were not confirmed by our results. However, with the current findings, it might suggest a potential over-compensation (inhibition) mechanism behind the remission of depression. This potential mechanism was in a same rationale with the stress resilience phenomenon, which confirmed that an upgraded hyperpolarization-activated current (Ih) in a mild degree resulted in vulnerability, while an even larger Ih led to resilience instead of vulnerability [40].

Within the CEN (DLPFC seed) as well as between the DLPFC seed and DMN, the dissociation between the function connectivity of frontal cortex and that of parietal cortex between the current and remitted patients suggested that these two cortexes played different roles in the remission process of depression. Specifically, the similar lower connectivity pattern between the current and remitted depression in frontal cortex indicated that the disturbed frontal connectivity might be a trait like neural basis of depressive episode, which might be a stable predictor of high recurrence of depression [22]. In contrast, the unique mode of lower connectivity in parietal cortex in the remitted patients suggested that the decreased parietal connectivity might be a reliable neural biomarker of remission [25)]. Indeed, previous study has reported that twoweek antidepressant administration in healthy volunteers reduces connectivity between DMN and task-positive network (very similar with CEN) [41], which is consistent with our results. The knowledge, as far as we know, is among the first to observe the function connectivity between DMN and CEN in the remitted depression, which helps to uncover the neural mechanism during the remission of depression.

Moreover, a correlation between functional connectivity and the clinical variables of patients was conducted, which indicated that longer duration of illness of MDD patients was correlated with decreased functional connectivity between DLPFC seed and left parietal cortex, and more episodes were correlated with decreased functional connectivity between DLPFC seed and right superior medial frontal cortex. Higher BDI scores (severe depression) were correlated with increased functional connectivity between mPFC seed and left medial frontal cortex. In contrast, longer duration of illness of RMD patients was correlated with 


\section{Default Model Network Mechanism in Current and Remitted Depression Research}

decreased functional connectivity between DLPFC seed and frontal cortex, and more episodes were correlated with elevated functional connectivity between DLPFC seed and frontalparietal cortex, as well as between mPFC seed and parietal cortex. Similar with MDD patients, higher BDI scores were correlated with increased functional connectivity between $\mathrm{mPFC}$ seed and right medial frontal cortex. The results suggested that depressive episodes were correlated with increased DMN activity and decreased DLPFCDMN connectivity [17], while depression remission was correlated with elevated DLPFCDMN connection, as well as CEN activity. Our findings indicated that elevated DMN activity might be a stable marker of occurrence or recurrence of depression, decreased DLPFCDMN connectivity was a marker of depressive episode, while increased DLPFC-DMN connectivity and CEN activity was related to remission of depression. The knowledge, as far as we know, is among the first to reveal the relationship between clinical variables and function connectivity in the current and remitted depression, which helps to offer reliable neural markers for clinical treatment of depression.

Limitation: The present study had two limitations. Firstly, the intelligence level of participants was not assessed, which might influence the results in a sense. Secondly, patients of current depression were medicated, which might influence the behavioral or neural response. However, previous research indicated no significant influence of medication on behavioral or neural response [42].

In conclusion, the present study offers a first experimental evidence of function connectivity differences between DMN and CEN in the current and remitted depression, which helps to uncover the neural mechanism during the process of depression remission. Specifically, the different connectivity mode in parietal cortex between the current and remitted depression emphasizes the function of parietal cortex in the remission of depression, which might be a reliable biomarker of remission. The similar connectivity pattern in the frontal cortex between the current and remitted depression hints a trait like neural basis of depressive episode, which might be a stable predictor of recurrence. The lower function connectivity within the DMN in remitted depression suggests a potential over-compensation (inhibition) mechanism behind the remission of depression. Our results offer important neural biomarkers for clinical treatment of depression, which could be practiced and tested in the future.

\section{Acknowledgement}

Doctor Dai claimed that this study was supported by National Social Science Fund of China (17XSH001), the innovation project of People's Liberation Army of China (17QNP008), and the key project of Chongqing education fund (162061). The authors thanked clinical doctors who devote to patients recruitment, including Dr. Dr. L. D. Hu from Geleshan Psychiatry Hospital, Dr. X. Y. Zhou from Psychiatry center of Chongqing, Dr. $Y$. He from 324 Hospital of Chong Qing. We appreciated the endeavor of all graduate students who participated this study as research assistants. We also thanked all subjects who took part in this study, especially the clinical patients.

\section{References}

1. Smith K. Mental health: A world of depression. Nature 515(1), 180-1801 (2014).

2. Mathews A, MacLeod C. Cognitive vulnerability to emotional disorders. Annu. Rev. Clin. Psychol 1(1), 167-195 (2005).

3. Taylor MJ. Non-spatial attentional effects on P1. Clin. Neurophysiol 113(1), 1903-1908 (2002).

4. De Raedt R, Koster EHW. Understanding vulnerability for depression from a cognitive neuroscience perspective: $A$ reappraisal of attentional factors and a new conceptual framework. Cogn. Affect. Behav. Neurosci 10(1), 50-70 (2010).

5. Koster EHW, De Lissnyder E, De Raedt R. Rumination is characterized by valence-specific impairments in switching of attention. Acta. Psychologica 144(3), 563-70 (2013).

6. Zhu X, Zhu Q, Shen H, et al. Rumination and Default Mode Network Subsystems Connectivity in First-episode, Drug-Naive Young Patients with Major Depressive Disorder. Sci. Rep 7(1), 43105 (2017).

7. O'Reardon JP, Solvason HB, Janicak PG, et al. Efficacy and safety of transcranial magnetic stimulation in the acute treatment of major depression: a multisite randomized controlled trial. Biol. Psychiatry 62(1), 1208-1216 (2007).

8. Haber SN, Knutson B. The reward circuit: Linking primate anatomy and human imaging. Neuropsychopharmacology 35(1), 4-26 (2010).

9. Fox MD, Raichle ME. Spontaneous fluctuations in brain activity observed with functional magnetic resonance imaging. Nature.
Reviews. Neuroscience 8(1), 700-711 (2007)

10. Zhong $X, P u$ WD, Yao SQ. Functional alterations of fronto-limbic circuit and default mode network systems in first-episode, drugnaïve patients with major depressive disorder: A meta-analysis of resting-state $\mathrm{fMRI}$ data. $J$. Affect. Disord 206(1), 280-286 (2016).

11. Mulders PC, van Eijndhoven PF, Pluijmen J, et al. Default mode network coherence in treatment-resistant major depressive disorder during electroconvulsive therapy. J. Affect. Disord 205(1), 130-7 (2016).

12. Shirer WR, Ryali S, Rykhlevskaia E, et al. Decoding subject-driven cognitive states with whole-brain connectivity patterns. Cerebral. Cortex 22(1), 158-165 (2012).

13. Salehinejad MA, Ghanavai E, Rostami R, et al. Cognitive control dysfunction in emotion dys- 
regulation and psychopathology of major depression(MD): Evidence from transcranial brain stimulation of the dorsolateral prefrontal cortex (DLPFC). J. Affect. Disord 210(1), 241-248 (2017).

14. Chen AC, Oathes DJ, Chang C, et al. Causal interactions between fronto-parietal central executive and default-mode networks in humans. Proc. Natl. Acad. Sci. U.S.A 110(1), 19944-19949 (2013).

15. Fox MD, Snyder AZ, Vincent JL, et al. The human brain is intrinsically organized into dynamic, anticorrelated functional networks. Proc. Natl. Acad. Sci. U.S.A 102(1), 9673-9678 (2005).

16. Chang CE, Glover GH. Effects of model-based physiological noise correction on default mode network anti-correlations and correlations. Neuroimage 47(1), 1448-1459 (2009).

17. Liston C, Chen AC, Zebley BD, et al. Default mode network mechanisms of transcranial magnetic stimulation in depression. Biolog. Psychi 76(7), 517-526 (2014).

18. Posner J, Cha J, Wang Z, et al. Increased default mode network connectivity in individuals at high familial risk for depression. Neuropsychopharmacology 41(7), 1759-767 (2016).

19. Koenigs M, Grafman J. The functional neuroanatomy of depression: distinct roles for ventromedial and dorsolateral prefrontal cortex. Behav. Brain Res 201(2), 239-243 (2009).

20. Farb NA, Anderson AK, Bloch RT, et al. Mood-linked responses in medial prefrontal cortex predict relapse in patients with recurrent unipolar depression. Biol. Psych 70(4), 366-72 (2011).

21. Gusnard DA, Akbudak E, Shulman G, et al. Medial prefrontal cortex and self-referential mental activity: Relation to a default mode of brain function. Proc. Natl. Acad. Sci. U.S.A 98(1), 4259-4264 (2001).

22. Zhang B, Li S, Zhuo C, et al. Altered task-specific deactivation in the default mode network depends on valence in patients with major depressive disorder. J. Affect. Disord (2016).

23. Jeon HA, Friederici AD. Degree of automaticity and the prefrontal cortex. Trends. Cogn. Sci 19(5), 244-250 (2015).

24. Kito S, Hasegawa T, Koga Y. Cerebral blood flow ratio of the dorsolateral prefrontal cortex to the ventromedial prefrontal cortex as a potential predictor of treatment response to transcranial magnetic stimulation in depression. Brain. Stimulation 5(4), 547-553 (2012).

25. Bartova L, Meyer BM, Diers K, et al. Reduced default mode network suppression during a working memory task in remitted major depression. J. Psychiatr. Res 64(1), 9-18 (2015).

26. Schiller CE, Minke IJ, Smoski MJ, et al. Remitted major depression is characterized by reduced prefrontal cortex reactivity to reward loss. J. Affect. Disord 151(2), 756-762 (2013).

27. Wu D, Yuan Y, Bai F, et al. Abnormal functional connectivity of the default mode network in remitted late-onset depression. J. Affect. Disord 147(1-3), 277-287 (2013).

28. Yin $Y$, Wang Z, Zhang Z, et al. Aberrant topographical organization of the default mode network underlying the cognitive impairment of remitted late-onset depression. Neuroscience. Letters 629(1), 26-32 (2016).

29. Kroenke K, Spitzer RL, Williams JBW. The PHQ-9:Validity of a Brief Depression Severity Measure. J. Gen. Intern. Med 16(1), 606-613 (2001).

30. Beck AT, Steer RA, Brown GK. Manual for the Beck Depression Inventory. San Antonio, TX: The. Psychological. Association (1996).

31. Hamilton M. A rating scale for depression. Journal of Neurological and Neurosurgical Psychiatry. 1960;23:56-62.

32. APA. APA Summary of Practice-Relevant Changes to the DSM-IV-TR. Washington: American Psychiatric Association (2000).

33. de Graaf LE, Gerhards SA, Arntz A, et al.
One-year follow-up results of unsupported online computerized cognitive behavioural therapy for depression in primary care: $\mathrm{A}$ randomized trial. J Behav Ther Exp Psychiatry 42(1), 89-95 (2011).

34. Dai Q, Feng ZZ. Deficient Interference Inhibition for Negative Stimuli in depression: An event-related potential study. Clin. Neurophysiol 122(1), 52-61 (2011).

35. Liu P, Shu L. Beck Depression Inventory, BDI; Beck Anxiety Inventory, BAl; Hamilton Depression Rating Scale, HDRS (Chinese). Chin Jour of Men Hea (1999).

36. Xu Y, Wu HS, Xu YF. The reliability and validity analysis: Application of patient health questionnaire - 9 in commility elders (Chinese). Pychiatry. Medcine. Of. Shanghai 19(5), $257-259$ (2007).

37. Beck AT, Epstein N, Brown G, et al. An inventory for measuring clinical anxiety: psychometric properties. J. Consult. Clin. Psychol 56(1), 893-897 (1988).

38. Song XW, Dong ZY, Long XY, et al. REST: A Toolkit for Resting-State Functional Magnetic Resonance Imaging Data Processing. PLOS. ONE 6(9), e25031 (2011).

39. Downar J, Geraci J, Salomons TV, et al. Anhedonia and reward-circuit connectivity distinguish nonresponders from responders to dorsomedial prefrontal repetitive transcranial magnetic stimulation in major depression. Biolog. Psychi 76(3), 176-185 (2014).

40. Johnson J, Panagioti M, Bass J, et al. Resilience to emotional distress in response to failure, error or mistakes: A systematic review. Clin. Psychol. Rev 52(1),19-42 (2017).

41. van Wingen GA, Tendolkar I, Urner M, et al. Short-term antidepressant administration reduces default mode and task-positive networkconnectivity in healthy individuals during rest. Neuroimage 88(1), 47-53 (2014).

42. Baert S, De Raedt R, Schacht R, et al. Attentional bias training in depression, Therapeutic effects depend on depression severity. J. Behav. Ther. Exp. Psychiatry 41(1), 265-274 (2010). 